\title{
O FAMILIAR ACOMPANHANTE COMO ESTÍMULO COMPORTAMENTAL DE PACIENTES INTERNADOS EM TERAPIA INTENSIVA
}

\author{
Family companionship as a behavioural stimulus for intensive care patients \\ El acompañante familiar como dispositivo para la estimulación de pacientes internados \\ en cuidadosintensivos
}

Verônica Lopes Louzada Vidal', Sílvia Teresa Carvalho de Araújo ${ }^{2}$, Michel Perreault ${ }^{3}$ e Albert Lengruber de Azevedo ${ }^{4}$

Recebido em 04/05/2012,reapresentado em 31/08/2012 e aprovado em 30/11/2012

\begin{abstract}
Resumo
0 objetivos deste estudo foram levantar as expressões verbais e não verbais presentes no comportamento do paciente antes, durante a inserção e permanência do familiar acompanhante no centro de terapia intensiva, e analisar comparativamente as alterações comportamentais do paciente durante esses momentos da internação. Método: 0 cenário foi o centro de terapia intensiva de um hospital no Rio de Janeiro, conveniado com o Sistema Único de Saúde (SUS). A abordagem qualitativa resultou da observação livre sobre o comportamento de cinco pacientes e registros em diário de campo durante três dias consecutivos. Priorizou-se observação do paciente no primeiro dia de internação e após a inserção ininterrupta do familiar acompanhante por três dias consecutivos. Resultados: As mudanças nas reações verbais e não verbais do paciente após os estímulos gerados pela presença do familiar variaram de ausência de fala e movimentos, passividade no agir, a reações de aceitação dos procedimentos, comunicação cinésica ampliada e maior participação no cuidado.
\end{abstract}

Palavras-chave: Enfermagem, Comportamento, Centro de Terapia Intensiva.

\begin{abstract}
Objectives: record patients' verbal and nonverbal expressions and behavior when they were unaccompanied, and later when a family companion was present and comparatively analyze the alterations in patient behavior observed during these periods of hospitalization. Method: The study was conducted at the intensive care unit of a hospital in Rio de Janeiro, which accepts patients from the Brazilian unified health system (SUS). A qualitative approach was taken, involving free observation and daily record keeping in field diaries of five patients' behavior during three consecutive days. We prioritized the observation of patients on the first day of hospitalization and subsequently when they had continuous family companionship for three consecutive days. Results: The family companion served as a stimulus for positive changes in the patients' verbal and nonverbal reactions, from of a lack of speech and movement and general passivity, to reactions that showed an acceptance of procedures, increased kinetic communication and greater participation in care.
\end{abstract}

Keywords: Nursing, Behavior, Intensive Care Unit.

\section{Resumen}

Objetivos: identificar las expresiones verbales y no verbales presentes en el comportamiento del paciente antes y durante la inserción y permanencia del familiar acompañante en el centro de terapia intensiva y analizar comparativamente las alteraciones comportamentales del paciente durante eses momentos de la hospitalización. Método: el escenario fue un centro de terapia intensiva de un hospital en Rio de Janeiro, convenio firmado con el Sistema Único de Salud (SUS). El enfoque cualitativo resultó de la observación libre sobre el comportamiento de cinco pacientes y de los registros en diario de campo durante tres días consecutivos. Se ha dado prioridad a la observación del paciente en el primer día de hospitalización y después de la inserción ininterrumpida del familiar acompañante por tres días consecutivos. Resultados: los cambios en las reacciones verbales y no verbales del paciente después de los estímulos generados por la presencia del familiar cambiaron desde la ausencia de habla y movimientos y pasividad en el actuar, para reacciones de aceptación de los procedimientos; comunicación cinésica ampliada y mayor participación en el cuidado.

Palabras claves: Enfermería, Conducta, Unidades de Cuidados Intensivos

\footnotetext{
${ }^{1}$ Mestre em Enfermagem pela Escola de Enfermagem Anna Nery, da Universidade Federal do Rio de Janeiro (UFRJ), Enfermeira Intensivista. Docente e coordenadora do curso de Enfermagem do Centro Universitário de Barra Mansa - RJ. Brasil. E-mail: veronica.vidal@oi.com.br;

${ }^{2}$ Doutora em Enfermagem. Professora Associada II do Departamento de Enfermagem Médico-Cirúrgica (DEMC), da Escola de Enfermagem Anna Nery, da Universidade Federal do Rio de Janeiro. Doutora em Enfermagem. Rio de Janeiro - RJ. Brasil. E-mail stcaraujo@gmail.com;

${ }^{3} \mathrm{PhD}$ em Sociologia. Docente da Universidade de Montreal no Canadá, Prof. Visitante 2006 a 2010 na Escola de Enfermagem Anna Nery, da Universidade Federal do Rio de Janeiro. Montreal - Canadá. E-mail: direção@eean.ufrj.br;

${ }^{4}$ Enfermeiro, Especialista em Enfermagem do trabalho e Especialista em Estratégia de Saúde da Família. Responsável Técnico no Hospital Psiquiátrico Santa Alice, RJ, Supervisor de Enfermagem no Hospital Psiquiátrico - Instituto Doutor Francisco Spínola, Rj, mestrando da Escola de Enfermagem Anna Nery/UFRJ. Membro do grupo de pesquisa Comunicação em Enfermagem Hospitalar: Clientes de Alta Complexidade (CEHCAC/NUPENH). Rio de Janeiro

- RJ. Brasil. E-mail:albertenfermagem@yahoo.com.br
} 


\section{INTRODUÇÃO}

Este artigo visa apontar alguns resultados da dissertação de mestrado apresentada à Escola de Enfermagem Anna Nery/UFRJ, desenvolvida em Unidade de Terapia Intensiva de um Hospital no interior do município do RJ. Tem como objetivos levantar as expressões verbais e não verbais presentes no comportamento do paciente antes, durante a inserção e permanência do familiar acompanhante no centro de terapia intensiva, e analisar comparativamente as alterações comportamentais do paciente durante esses momentos da internação.

Em geral, os pacientes internados em terapia intensiva sofrem com a doença e com a lesão cujo comprometimento gerou a necessidade do cuidado; eles ficam privados do contato familiar, seu único elo afetivo; e ainda ficam expostos a um ambiente estranho, convivendo com profissionais que atuam maciçamente com técnicas e procedimentos invasivos, em função das doenças e dos tratamentos necessários. 0 processo invasivo ligado ao avanço tecnológico necessário como complemento terapêutico também gera mudanças significativas na experiência da hospitalização.

As condições ambientais de um setor fechado impõem confinamento, falta de informações e isolamento social, e ainda determinam que os pacientes fiquem privados sensorialmente de estímulos familiares. Comumente, apresentam delírios, alucinações, psicoses, ansiedades, medo e depressão resultantes deste fenômeno hospitalar. São ambientes descritos na literatura científica como aqueles em que os pacientes são privados de estímulos sensoriais normais, mas são a cada segundo de sua permanência neste setor bombardeados com outros, estranhos e contínuos, ausentes no seu ambiente doméstico ${ }^{1}$.

Acreditávamos que a inserção dos familiares acompanhantes em unidade de terapia intensiva poderia contribuir para minimizar a instabilidade emocional do paciente e familiar acompanhante, resultando em melhora do equilíbrio e de maior participação do paciente nos cuidados, durante a internação. Identificadas pela enfermeira por meio do cuidado vigilante e observador, focamos nossa investigação para levantar as principais alterações das expressões não verbais do paciente, de forma comparativa antes, após inserção e durante a permanência do familiar acompanhante.

Esta pesquisa quer respeitar as diretrizes gerais da Política Nacional de Humanização² que no Brasil coloca o dispositivo do familiar acompanhante nos diferentes níveis de atenção. Nesta política destacam-se como diretrizes: adequar os serviços à cultura local, respeitando a privacidade e promovendo uma ambiência acolhedora e confortável, e garantir a presença do acompanhante e de sua rede social, em âmbito hospitalar.

O Brasil parece ser o único país com sistema de saúde que se utiliza do dispositivo de familiar acompanhante como meta central de humanização do cuidado. Este dispositivo existe fora do Brasil no campo do parto, onde resultados positivos são cada vez mais documentados. Nos outros setores da saúde, esse dispositivo não parece existir e, mesmo quando o cuidado é dito centrado no paciente, algumas pesquisas discorrem sobre ligações entre equipe profissional e o cuidado e/ou sobre os familiares do paciente.

Segundo uma revista de literatura cientifica internacional, nem existe um dispositivo de familiar acompanhante, como é o caso no Brasil dentre todos os serviços das instituições de saúde, e como é o caso, em geral, no campo bem particular do parto ${ }^{3}$. E não existe qualquer forma de avaliação da contribuição do familiar acompanhante sobre 0 estado de saúde dos pacientes acompanhados, o que é particularmente o caso na terapia intensiva.

Em 21 ensaios envolvendo 15.061 mulheres que preencheram os critérios de inclusão, com resultados de análises de efeitos aleatórios, salvo indicação em contrário, observouse que as mulheres atribuídas para o apoio contínuo eram mais propensas a ter um parto vaginal espontâneo e menos propensas a ter analgesia intraparto. Além disso seus trabalhos eram mais curtos, elas eram menos propensas a ter uma cesariana ou parto vaginal instrumental. 0 apoio contínuo foi mais eficaz quando feito por uma mulher que nem fazia parte da equipe do hospital, em ambientes em que a analgesia epidural não estava rotineiramente disponível ${ }^{3}$.

Nos outros setores da saúde, o dispositivo de inserção do familiar não parece existir e, mesmo quando o cuidado é dito centrado no paciente, existem algumas pesquisas sobre ligações entre equipe profissional, o cuidado e os familiares do paciente, mas os efeitos não são medidos. Embora na área de psicologia, o nível comportamental da análise científica considera 0 organismo a base a partir da qual as relações funcionais entre o comportamento e os eventos ambientais, que geram ansiedade, são experimentalmente examinadas ${ }^{4}$.

Em um revisão sistemática não foi encontrado estudo científico que avaliasse os efeitos da família no cuidado centrado à crianças hospitalizadas, quando comparado aos modelos standard ou profissional de prestação de cuidados, em resultados de serviços à criança, família e saúde, mesmo se este modelo centrado na família estivesse cada vez mais proeminente no cuidado. E concluiu-se que um exame muito mais rigoroso, com o cuidado centrado na família, como um modelo para o atendimento às crianças e famílias nos serviços de saúde, é necessário 5 .

No campo da terapia intensiva, o apoio social aos cuidados intensivos estava voltado ao apoio para os cuidadores ${ }^{6}$. Concluiu-se que não há evidências de que intervenções de apoio a cuidadores informais de doentes em fase terminal podem ajudar a reduzir o estresse psicológicos dos cuidadores. Estes achados sugerem que os médicos devem inquirir sobre as preocupações dos cuidadores, considerando que eles podem beneficiar o apoio adicional. Os autores não encontraram 
resultados nos estudos sobre o efeito dos cuidadores sobre a saúde dos pacientes.

Precisamos entender as mudanças fisiológicas, as oscilações nas reações comportamentais (respondentes, operantes, verbais, não verbais), pois resultam da ansiedade e afetam a terapia verbal dos indivíduos em condições de hospitalização, pois sua condição emocional, de enfrentar, ou não, os novos desafios durante a internação estará expressa no seu comportamento. Envolvem a referência a componentes fisiológicos, condicionamento operante e respondente, direto e indireto, não verbal, além de relações com operações facilitadoras de bem-estar, ou mal-estar presentes no contexto?

A exposição do organismo a estímulos aversivos e préaversivos, controláveis ou incontroláveis, produz uma condição fisiológica particular, concomitante a uma mudança no responder geral do organismo. Portanto, a ansiedade é um estado corporal produzido por contingências de reforçamento específicas: um estímulo sinaliza a apresentação de um estímulo aversivo e não há comportamento de fuga-esquiva possível ${ }^{7}$. A alteração fisiológica produzida pelas contingências que explicam a ansiedade é abordada salientando-se suas funções em uma relação comportamental.

Há, no entanto, a necessidade de mais pesquisas para explorar os benefícios identificados na inserção do familiar acompanhante, e para avaliar os efeitos dessa intervenção na saúde física e emocional do paciente. Ensaios precisam relatar como os códigos de comportamento podem garantir a especificação de novas regras a serem apreendidas por todose que afetará o comportamento de cada um. Lembrando Charles Darwin, a predição e o controle não terão sucesso se o fenômeno a ser previsto e controlado não for adequadamente descrito ${ }^{4}$.

\section{METODOLOGIA}

Realizou-se um estudo descritivo, cuja finalidade foi observar, descrever e documentar os aspectos de uma dada situação, com abordagem qualitativa. Desta forma, priorizamos trabalhar com 0 universo de significados, motivos, aspirações, crenças, valores e atitudes, o que corresponde a um espaço mais profundo das relações, dos processos e dos fenômenos que não podem ser reduzidos à operacionalização de variáveis ${ }^{8-9}$. Esta abordagem torna possível, então, responder a questões do indivíduo no ambiente de cuidado intensivo com um nível de realidade que não pode ser somente quantificado.

A produção dos dados foi realizada no ano de 2009, em hospital localizado no município, interior do Estado do Rio de Janeiro, conveniado com o Sistema Único de Saúde (SUS). Entretanto, realiza também atendimentos junto à rede privada, dispondo a unidade de terapia intensiva de 15 (quinze) leitos, sendo 08 (oito) para os atendimentos reservados aos pacientes coronarianos e 07 (sete) para atendimentos dos pacientes da clínica geral.
A investigação foi realizada após aprovação não só do projeto de dissertação pelo Comitê de Ética e Pesquisa da Escola de Enfermagem Anna Nery e Hospital São Francisco de Assis, sob Protocolo $n^{0}$ 08, mas também, da direção do hospital, da coordenação da enfermagem, da chefia médica e de enfermagem do setor.

0 contato pessoal com o familiar do paciente internado foi autorizado pela chefia de enfermagem e o convite de participação foi estabelecido por meio do termo de consentimento, durante a segunda visita institucional no setor. E, para assegurar a espontaneidade durante a interação, esclarecemos apenas que a investigação era sobre comunicação, mas não detalhamos no termo como se daria o processo de observação.

Como a proposta inicial aos acompanhantes era sua inserção no acompanhamento do paciente familiar internado na terapia intensiva, de maneira consecutiva e integral, essa oportunidade e benefício foram aceitos e autorizados formalmente por ele, por meio do Termo de Consentimento Livre e Esclarecido, conforme dispõe a Resolução nº 196 do Conselho Nacional de Saúde.

Preparamos o ambiente com poltrona confortável e divisória, e preparamos a equipe de enfermagem para a inserção do familiar no setor. A observação e a inserção do vínculo familiar na unidade de terapia intensiva foram planejadas para acontecer com apenas um paciente de cada vez, nos meses de fevereiro a maio. Assegurando sua permanência no setor até a transferência, alta ou óbito do paciente.

Priorizamos para essa análise os dados produzidos apenas nos quatro primeiros dias de internação. 0 estudo de caso ${ }^{9}$ permitiu a observação e os registros em diário campo, relativos ao comportamento do paciente antes e após inserção do familiar acompanhante. 0 s registros emergentes durante 0 cuidado de enfermagem prestado permitiu pontuar uma variedade de dados qualitativos sobre a comunicação não verbal e as variações dessas manifestações no comportamento do paciente.

Na realidade, os estudos de caso frequentemente estão preocupados com a compreensão da natureza da intervenção, tanto quanto no estabelecimento de seus custos e efeitos. E, para assegurar a observação não participante, contamos com uma enfermeira com experiência na clínica e treinada, para participar como auxiliar de pesquisa, que procedeu aos registros em diário de campo.

Ainda que o paciente e familiar não tenham sido esclarecido sobre o processo de investigação, consideramos prioridade de produção de dados e análise às observações sobre o comportamento não verbalizado do paciente sem acompanhante, aqueles evidenciados durante as interações com o familiar e as expressões do paciente ao longo do cuidado de enfermagem.

Vale destacar que, assim como na metodologia assistencial de enfermagem, os aspectos objetivos e subjetivos 
do paciente foram considerados a partir da clínica e do cuidado. E a comunicação não verbal do paciente na internação é valorizada como aspecto subjetivo essencial para a compreensão do comportamento indicativa de necessidade a ser atendida pelo e no cuidado.

Como nos estudos de observação, o pesquisador tem flexibilidade de considerar as várias dimensões importantes, que pode estar sobre eventos amplamente definidos, como as alterações de humor do paciente, ou em detalhes comportamentais altamente específicos, como gestos e expressões faciais, ${ }^{10}$ confirmando a finalidade dos estudos descritivos já mencionados.

A inserção do familiar junto ao paciente em unidade de terapia intensiva nos mostrou a condição de interação significativa e motivadora a partir do vínculo e do acompanhamento durante a internação. A análise das descrições permitiu a pesquisadora classificar os dados a partir da tipologia da comunicação não verbal.

\section{APRESENTAÇÃO DOS RESULTADOS}

Os dados foram coletados junto a 05 (cinco) pacientes, antes e durante a inserção do familiar acompanhante, constituindo-se da observação das reações manifestadas após os estímulos gerados pela presença do familiar. Trata-se, portanto, de observação sobre o alcance do paciente e do familiar-acompanhante. 0 campo da pesquisa dispõe de uma área circular fechada com vidros, que permite à equipe ter uma visão globalizada dos pacientes sob seus cuidados.

A escolha dos sujeitos obedeceu aos seguintes critérios de inclusão: ser paciente em ventilação invasiva ou não invasiva, avaliada na escala de sedação de 2 a 7 , responsivo aos estímulos verbais ou táteis; e de exclusão: ser paciente comatoso, sedação 1 , resposta mínima ou ausente aos estímulos nociceptivos ${ }^{1}$. Os critérios adotados evitaram o risco de comprometimento da fidedignidade dos dados coletados.

Os sujeitos que integraram a pesquisa apresentavam o seguinte perfil:

Paciente $n^{0}$ 1: $A A, 15$ anos, sexo feminino, sepse.

Paciente $n^{\circ}$ 2: $A X, 48$ anos, sexo masculino, aneurisma cerebral.

Paciente $n^{0}$ 3: SC, 31 anos, sexo feminino, pósoperatório com obstrução Intestinal.

Paciente $n^{0}$ 4: LVP, 29 anos, sexo masculino, neurocirurgia.

Paciente $n^{0}$ 5: SV, 65 anos, sexo feminino, hipertensão pulmonar.

A partir do processo de observação dos pacientes hospitalizados na unidade de terapia intensiva, foi possível formular duas categorias analíticas e cinco subcategorias. A primeira categoria intitulada reações físicas, psíquicas e emocionais na ausência do familiar-acompanhante resultou nas seguintes subcategorias: ausência de fala e movimentos e passividade no agir e no aceite dos procedimentos; alterações na percepção sensorial.

A segunda categoria intitulada reações físicas, psíquicas e emocionais na presença do familiar-acompanhante geraram as subcategorias: comunicação ampliada, com a equipe e 0 familiar; movimentos ativos do corpo e percepções sensoriais.

Após as observações realizadas e análise dos dados, tornou-se evidente que a presença do familiar acompanhante trouxe mudanças positivas no comportamento do paciente, que se mostrou mais confor tável e estável emocionalmente no seu quadro clínico. Pudemos concluir na análise comparada entre os dados apresentados a observação dos pacientes sem familiar acompanhante e a observação dos mesmos pacientes com familiar acompanhante.

Pudemos concluir mudanças significativas na análise comparada entre os dados apresentados, a observação dos pacientes sem familiar acompanhante e a observação dos mesmos pacientes com familiar acompanhante. Os pacientes manifestam-se não verbalmente de maneira mais incisiva, intensa, frequente e mais clara na presença do familiar. Também participam ativamente dos cuidados após a inserção do mesmo.

Na primeira experiência, sem familiar acompanhante: 0 paciente, com sepse, permanecia com olhos fechados, às vezes chorava silenciosamente; mantinha-se imóvel no leito; dizia que a sonda doía; não dormia, pois sentia medo; não reclamava, era literalmente paciente. Já com o familiar acompanhante, a paciente queria ficar sentada assistindo TV; ficava segurando a mão da tia com medo de ela ir embora; sorria sempre para a equipe; repousava bem; os seus banhos começavam a ser no chuveiro; mantinha-se sentada no leito e já interagia bastante com a equipe.

Outro sujeito observado, com aneurisma cerebral era desanimado, inexpressivo, só interagia com abertura ocular quando solicitado pela equipe, não manifestava movimentos corporais, mostrava-se inseguro e demonstrava dor durante os movimentos realizados pela equipe, pelas expressões faciais, lábios rígidos e musculatura facial tensa. Na presença de seu familiar já tentava conversar; piscava os olhos nas respostas afirmativas. Mesmo entubado, sorria e seus olhos lacrimejavam.

No pós-operatório de obstrução intestinal, outra paciente se mantinha quieta, imobilizada no leito, com comunicação limitada, desânimo, preocupação, olhar parado e perdido, nunca sorria, não dormia à noite, não manifestava desejo de levantar do leito ou deambular. Após inserção do familiar, durante 0 banho no leito, já virava o corpo para ajudar na mobilização; conversava; sentou-se na poltrona; trocou sorriso com a mãe; interou-se mais com a equipe; relatou ter dormido bem; manteve-se ativa e falante; recusou-se voltar ao leito.

Outra paciente de neurocirurgia apresentava comunicação restrita a pequenos gestos, só abria os olhos com insistência da equipe, imobilizada no leito, demonstrava-se sonolência, com face inexpressiva e sem movimentos ativos. Após inserção do familiar, os dados objetivos, mensuráveis pela 
monitorização, oscilavam. Sempre que a esposa interagia com o paciente, o carinho dispensado através do toque e da conversa com entonação de voz tranquila e firme gerava alteração significativa nos valores de pulsação, elevando entre 10 e 15 os batimentos cardíacos por minuto e com melhora da saturação de oxigênio em 0,5\% a 0,8\%.

Apresentando hipertensão pulmonar outra participante da pesquisa não manifestava nenhum sinal de interação com o meio, sem abertura ocular, com restritas reaç̃̃es aos estímulos, apresentava alteração na face quando era aspirada, mordia o tubo orotraqueal, tinha movimentos restritos de membros inferiores e superiores. Após inserção do familiar, a paciente olhava fixo para a filha tentando se comunicar; abertura ocular espontânea; sempre segurando a mão da filha; movimentava os lábios; colocou uma perna sobre a outra; dormia de forma mais tranquila; segurava-lhe a mão.

Como fato relevante houve também mudança na postura da equipe de enfermagem, cujo olhar passou a ser diferenciado em relação ao cuidado prestado, mesmo com a inclusão do acompanhante familiar em tempo integral à beira do leito do paciente durante três dias consecutivos. No início da pesquisa, muitos reagiram a essa mudança no setor, mas, durante a inserção do familiar acompanhante, a equipe pode reconhecer a importância da preservação dos vínculos familiar e social durante a realização terapêutica de suas ações assistenciais.

\section{DISCUSSÃO}

Os dados de pesquisa evidenciam, a partir das próprias demandas dos pacientes, as necessidades implícitas nas expressões não verbalizadas de cada um ${ }^{10} .0$ familiar serviu de alicerce já que houve alterações comportamentais do paciente diante dessa presença, demonstrando tranquilidade e maior participação ativa durante os cuidados.

0 conceito de ansiedade tem sido empregado na análise do comportamento e sob o controle de diferentes eventos ou relações. E os níveis de ansiedade variam quanto ao papel atribuído às alterações fisiológicas e são afetados pelas relações respondentes e operantes, verbais e não verbais. As variações de visões complementares de um fenômeno complexo, em que eventos adquirem diferentes funções a partir de processos de condicionamento direto e indireto e afetam a terapia verbal e o autocontrole do indivíduo ${ }^{7}$.

A inserção do familiar funcionou como estímulo positivo na redução da ansiedade pelas respostas não verbais, maior participação na interação, com melhor controle durante o cuidado. Entender o significado de um conceito, portanto, implica buscar as contingências das quais a resposta verbal ou não verbal específica do paciente nos parece ter sido em função do grande estímulo visual, tátil, sonoro, de aproximação e permanência ininterrupta de duas pessoas que se reconhecem com funções familiares e sociais.
0 problema da imprecisão subjetiva das expressões não verbais aparece na análise comparada entre os dois momentos da investigação. Essa foi pontuada pelo comportamento de inércia demonstrada pelo paciente antes da inserção do familiar e que foi diferente do apresentado após a sua inserção durante três dias ininterruptos.

A ênfase recai na força e na precisão que os sentidos corporais possuem como radares de percepções e que influencia direta e/ou indiretamente como estímulos positivos com melhores respostas adaptativas na internação e na interação do paciente com o familiar e os membros da equipe de enfermagem. Destacamos aspectos referentes à linguagem como fonte de controle de respostas e relações indiretas entre estímulos públicos e privados.

Portanto, há efeitos emocionais que podem ocorrer apenas quando um estímulo precede caracteristicamente um estímulo aversivo com um intervalo de tempo suficientemente grande para permitir a observação de mudanças comportamentais ${ }^{11}$. Assim, se o paciente tem medo de ser internado em unidade de terapia intensiva e se o estímulo verbal presente no cuidado da equipe de enfermagem desse setor acompanha seu pensamento porque é uma situação de verdade, real, então, o estímulo visual, sozinho, pode evocar uma relação emocional.

A falta de controle sobre eventos aversivos internos (fisiológicos) e externos (ambientais) desempenha um papel central no desenvolvimento e manutenção de muitos transtornos de ansiedade na terapia intensiva. Mudanças no responder operante não verbal podem ainda estar relacionadas a um reforçamento positivo ou negativo com responder ansioso $0^{11}$.

A ansiedade pode tanto ser mantida pela suspensão ou adiantamento de tarefas indesejáveis como reduzida pela oferta de atenção social ${ }^{12}$. A presença do familiar aumentou a motivação do paciente minimizando estímulos provocadores de ansiedade e de alterações fisiológicas no ambiente. A permanência do familiar ao lado do paciente foi importante no compartilhamento em momentos e contingências (aversivas) ambientais presentes e que são responsáveis pela instalação e manutenção da ansiedade ${ }^{12}$.

Na pesquisa, o nível de ansiedade do paciente esteve sob controle pela manutenção do familiar ao seu lado, que funcionou como estímulo reforçador positivo, pela relação de amparo e pela contingência de vínculo significativo favorecedor do autocontrole. A manutenção desse elo gerou reforçamento positivo e uma condição para manifestações corporais favoráveis, respondentes e operantes dos fenômenos emocionais.

As observações individualizadas e comparativas dos pacientes diante de cada gesto e dos movimentos antes e após a inserção de um familiar na UTI resultaram em mudanças significativas de comportamento e de recuperação rápida dos respectivos quadros clínicos. A sensibilidade expressa nas 
dimensões cognitivas, na abertura dos sentidos e na amplitude dos movimentos corporais dos pacientes ${ }^{10}$ intensificou a importância da comunicação verbal e não verbal no contexto de cuidado.

Temos buscado compreender os modelos assistenciais, como formas de organização da produção de serviços a partir de um determinado arranjo de saberes da área. Assim, entendemos que o campo de saberes e práticas da clínica é parte fundamental não só de tecnologias materiais para os instrumentos, mas também de tecnologias não materiais para o conhecimento técnico usado na produção da saúde. Nossas observações têm concluído que, para além dos instrumentos e conhecimento técnico, lugar de tecnologias mais estruturadas, há um outro, o das relações, que tem se verificado como fundamental para a produção do cuidado ${ }^{13}$.

0 trabalho em saúde é sempre relacional, porque dependente de trabalho vivo em ato, isto é, o trabalho no momento em que este está produzindo. Estas relações podem ser, de um lado, sumárias e burocráticas, em que a assistência se produz centrada no ato prescritivo, compondo um modelo que tem sua natureza, o saber médico hegemônico, produtor de procedimentos. Por outro lado, estas podem se dar como relações intersessoras e estabelecidas no trabalho em ato realizado no cuidado à saúde ${ }^{13}$.

Na UTI, o paciente necessita de cuidados que são baseados em uma visão holística da saúde individual e ecológica. Esta visão pode assegurar uma vigilância atenta da sua condição instável a fim de detectar e prevenir qualquer complicação, compreendendo e atendendo igualmente e com atenção na condição de ansiedade experimentada pelo paciente e sua família.

Considerando os cuidados da família, os resultados deste estudo destacam o fato de que os enfermeiros estão se afastando da família do paciente internado em unidade de terapia intensiva. Estes resultados de como equipe e família estabelecem comunicação também surgiram no estudo recente ${ }^{14}$ com as enfermeiras que trabalham em cuidados intensivos.

Nas entrevistas com 70 enfermeiros que trabalham em unidades de terapia intensiva, descobriu-se que apenas $47 \%$ dos enfermeiros às vezes conversam com a família do doente ou seguem o tratamento prescrito, $61 \%$ seguem as técnicas utilizadas e $20 \%$ deles nunca falam com a família sobre os sentimentos que ela vive ${ }^{14}$. Conclui-se que há uma falta geral de atividades de enfermagem na comunicação com familiares de pacientes.

$A$ atenção dada às famílias de pacientes com necessidade críticas é uma atitude empenhada na ética do cuidado; a assistência ao paciente em situação crítica será incompleta se os enfermeiros não enfrentarem a questão do sofrimento da família, pois ela desempenha um papel terapêutico útil sobre a enfermagem e é utilizadora dos serviços de saúde ${ }^{15}$. Estes são ideais éticos. Em outras palavras, esta pesquisa é a congruência da prática de enfermagem a partir de uma abordagem holística para a pessoa e sua família ${ }^{16}$.

Da mesma forma, a dimensão relacional do cuidado está melhorando em relação à família. Há um interesse pelos fatores que afetam a relação do enfermeiro com a família e as expectativas futuras a partir da perspectiva das enfermeiras que trabalham em unidade de terapia intensiva ${ }^{15}$. E o trabalho vivo é conceituado como 0 ato que pressupõe uma lógica relacional, tecnologias leves dependentes, integralidade da assistência e a operação das linhas de cuidado que garanta a atenção com implicação mútua ${ }^{13}$. Espera-se o reconhecimento de que ali há o encontro de sujeitos que juntos têm ação protagonista na produção da saúde e que precisam estar presentes nas diretrizes de intervenção/relação de acolhimento, estabelecimento de vínculo e responsabilização.

Refletindo coletivamente, os enfermeiros têm reconhecido a necessidade de mudar os aspectos da sua prática e dar mais atenção à família ${ }^{16}$. Inicialmente, eles consideravam a família como um fator externo à unidade e ao processo de atendimento ao paciente crítico, em seguida, tinham o contato com a família como causa do estresse; posteriormente ao processo reflexivo, eles mudaram e adotaram uma relação de confiança ou terapêutica.

A revisão da literatura de estudos relacionados às famílias na UTI durante os anos de 1993 a 2004 permitiram concluir que os enfermeiros estão preocupados com o grau de hostilidade com as famílias no ambiente de terapia intensiva e da gravidade das necessidades à medida que surgem ${ }^{17}$. No entanto, os estudos analisados usaram um projeto de pesquisa e não replicativo intervencionista, que é considerado necessário para implementar e avaliar sistematicamente o cuidado com foco específico na família.

Atributos próprios da relação humana são fundamentais na construção de vínculo entre a enfermeira, o paciente e a família durante cuidado. E, por isso, é necessário considerá-los como aspectos essenciais na relação entre seres humanos e no processo de cuidar. Isso implica considerarmos a expressão do sujeito e, também, a necessária manutenção da identidade do paciente no espaço social hospitalar, principalmente quando vivenciamos o cuidado de enfermagem ${ }^{18}$.

\section{CONCLUSÃO}

0 estudo com inserção do familiar foi uma investigação intervencionista, com uma análise preliminar que permite elaborar, implantar e avaliar novas estratégias de cuidados ao paciente e seu familiar, no contexto de cuidados críticos e em que o trabalho vivo deve ser entendido como potência real para mudanças significativas no modelo assistencial vigente.

Sendo assim, os resultados da pesquisa merecem um repensar dos profissionais sobre a importância do familiaracompanhante na UTI, durante a permanência do paciente nesta Unidade, diante dos benefícios que lhe foram propiciados de forma relevante nos aspectos comportamentais, emocionais e físicos. 
0 Brasil deveria ser uma referência de acolhimento nesta modalidade de visita aberta. Necessitamos de outros estudos para ampliar o conhecimento sobre seus efeitos na recuperação do paciente internado.

A presença do familiar acompanhante no contexto hospitalar nos permitiu repensar sobre a melhoria na regulação dos horários de visitas e dos períodos de permanência dos familiares acompanhantes junto aos pacientes durante a internação em unidade de terapia intensiva.

É necessário considerar as diversas relações interconectadas que constituem essa experiência para dar conta de compreender sua complexidade variável. Portanto, devemos considerar os aspectos comportamentais e sociais nas relações nos níveis filogenético, ontogenético e cultural.

Enfatizamos a necessária consciência profissional sobre a influência do ambiente e do contexto de terapia intensiva que afetam a condição corporal não verbal do paciente. A permanência do familiar na unidade permitiu a redução da ansiedade, o equilíbrio emocional e a restauração de manifestações não verbais ativas do paciente essenciais no cuidado e na sua recuperação.

\section{REFERÊNCIAS}

1. Cintra EA, Nishide VM, et al. Assistência de Enfermagem ao Paciente Gravemente Enfermo. 2. ed. Rio de Janeiro (RJ): Atheneu; 2001.

2. Ministério da Saúde (BR). Humaniza SUS: visita aberta e direito ao acompanhante. Ministério da Saúde. Secretaria de Atenção à saúde. Núcleo Técnico da Política Nacional de Humanização. 2. ed. Brasilia: Ministério da Saúde, 2007.

3. Hodnett ED, Gates S, Hofmeyr GJ, Sakala C, Weston J. Continuous support for women during childbirth. Cochrane Database Syst Rev. 2011 Feb 16;(2): CD003766. DOI: 10.1002/14651858.CD003766.pub3.

4. Glenn SS. Contingencies and metacontingencies: Toward e synthesis of behavior analysis and cultural materialism. Behav Anal. 1988; 11 (2): 161 79 .

5. Shields L, Pratt J, Davis L, Hunter J. Family-centred care for children in hospital. Cochrane Database Syst Rev. 2007; (1): CD004811.D0l: 10.1002/ 14651858.CD004811.pub2

6. Candy B, Jones L, Drake R, Leurent B, King M. Interventions for supporting informal caregivers of patients in the terminal phase of a disease. Cochrane Database Syst Rev. 2011; (6): CD007617. D0I: 10.1002/ 14651858.CD007617.pub2

7. Queiroz PP, Guilhard HJ. Identificação e análise de contingências geradoras de ansiedade: Caso clínico. In Guilhardi HJ, Madi MBBP. Queiroz PP, Scoz MC (Eds.), Sobre comportamento e cognição: Santo André, SP; ESETec, 2001; 7: 257-68.
8.Lakatos EV, Marconi MA. Metodologia científica. 4. ed. São Paulo (SP): Atlas; 2004

9.Pope C, Mays N. Pesquisa qualitativa na atenção à saúde. 3. ed. Porto Alegre (RS): Artmed; 2009.

10. Araújo STC. Os sentidos corporais dos estudantes no aprendizado da comunicação não verbal do cliente na recepção pré-operatória: uma semiologia da expressão através da sociopoética. [Tese]. Universidade Federal do Rio de Janeiro, Escola de Enfermagem Anna Nery; 2000.

11. Skinner, BF. Contingencies of reinforcement: A theoretical analysis. New York (USA): Appleton-Century-Crofts; 1969.

12.Zamignani, DR, \& Banaco, RA. Um panorama analítico-comportamental sobre os transtornos de ansiedade. Rev. bras. ter. comport. Cogn, 2005; 7: 77-92.

13. Merhy, EE. Saúde: A cartografia do trabalho vivo, 3. ed. São Paulo (SP): Editora Hucitec; 2002.

14. Zaforteza C Sánchez C, Lastra P. Análisis de la literatura sobre los familiares del paciente crítico: es necesario desarrollar investigación en cuidados efectivos. Enferm. intensiva. 2008; 19(2): 61-70.

15. Dallaire C, Dallaire M. Le savoir infirmier dans les fonctions infirmières. Dans C. Dallaire (Éd) Le savoir infirmier. Au cœur de la discipline et de la profession. 2008. Québec (CAN): Gaëtan Morin Éditeurs. p. 265-312.

16. Santana L, Yañez B, Martín J, Ramírez F, Roger I, Susilla A. Actividades de comunicación del personal de enfermería con los familiares de pacientes ingresados en una unidad de cuidados intensivos. Enferm. clín. 2009; 19(6): 335-9.

17. Johns C. Becoming a Reflective Practitioner. A reflective and holistic approach to clinical nursing, practice development and clinical supervision. London (ENG): Blackwell Science; 2000.

18.Ferreira MA, Figueiredo NMA, Arruda A, Alvim NAT. Cuidados fundamentais de enfermagem na ótica do cliente: uma contribuição para a Enfermagem Fundamental. Esc. Anna Nery Rev. Enferm. 2002; 6(3): 387-96. 